\title{
Comparing the Sensitivity of the MMPI-2 Clinical Scales and the MMPI-RC Scales to Clients Rated as Psychotic, Borderline or Neurotic on the Psychodiagnostic Chart
}

\author{
Robert M. Gordon ${ }^{1}$, Ronald W. Stoffey ${ }^{2}$, Bethany L. Perkins ${ }^{3}$ \\ ${ }^{1}$ Independent Practice, Allentown, USA \\ ${ }^{2}$ Psychology Department, Kutztown University, Kutztown, USA \\ ${ }^{3}$ Department of Education and Human Services, Lehigh University, Bethlehem, USA \\ Email: rmgordonphd@gmail.com
}

Received July 14 ${ }^{\text {th }}, 2013$; revised August $15^{\text {th }}, 2013$; accepted September $19^{\text {th }}, 2013$

\begin{abstract}
Copyright (C) 2013 Robert M. Gordon et al. This is an open access article distributed under the Creative Commons Attribution License, which permits unrestricted use, distribution, and reproduction in any medium, provided the original work is properly cited.
\end{abstract}

\begin{abstract}
The purpose of this study was to assess the differences between the MMPI-2 and the MMPI-RC scales in sensitivity to levels of psychopathology. Ninety-eight clients from forensic, disability and psychotherapy evaluations were evaluated on the MMPI-2 and RC scales and rated for personality organization (neurotic, borderline or psychotic) on the Psychodiagnostic Chart. The results over-all showed support that most of the MMPI-2 scales have more clinical sensitivity than the RC scales at all levels of psychopathology and particularly at the less pathological levels. K correction does not account for the elevation differences. Most of the RC scales add little to no incremental validity to the MMPI-2 Clinical scales except for RC 1, RC 2, and RC 9 and these may be used as supplemental scales.
\end{abstract}

Keywords: MMPI-2; MMPI-RC; MMPI-RF; Psychodiagnostic Chart; PDM; Psychopathology

\section{Introduction}

Tellegen, Ben-Porath, McNulty, Arbisi, Graham, and Kaemmer (2003) first produced the Restructured Clinical scales (RC) to improve measurement of the core constructs of the MMPI-2 Clinical scales, but then went on to develop a separate competing form of the MMPI. Rouse, Greene, Butcher, Nichols, and Williams (2008) looked at a sample of 78,159 respondents across diverse clinical settings and found that each RC scale was highly correlated with the existing MMPI-2 contents scales; higher, in fact, than the correlation between the RC scale and its parent scale concluding that the RC scales added nothing new to the MMPI-2 scales.

Binford and Liljequist (2008) compared the behavioral correlates of $\mathrm{RCd}, \mathrm{RC} 2$, and $\mathrm{RC} 4$ with their original Clinical scale counterparts (Scale 2, Scale 4), and conceptually related Content scales (DEP, ASP, CYN) in an outpatient clinical sample $(\mathrm{N}=150)$. The results indicated that $\mathrm{RC} 4$ is a stronger predictor of several antisocial behaviors than Clinical Scale 4 or the Content Scales ASP and CYN. In contrast, RC 2 demonstrated significantly lower correlations with several behaviors conceptually related to depression than its Clinical scale counterpart or DEP. Bolinskey, and Nichols (2011) concluded that $\mathrm{RC} 4, \mathrm{RC} 7$, and RC 9 are not necessarily equivalent to those assessed by the original scales.

The RC scales have higher internal consistency and discriminant validity than the original MMPI-2 clinical scales, which the authors believe, would lead to greater clinical utility. However, Gordon (2008) warned that the high internal consistency of the RC scales violates a basic assumption of complex psychopathology. Whereas some unitary symptoms such as anxiety or anger can be assessed with scales of high internal consistency, such homogenized scales will not work well for complex diagnostic conditions such as Hysteria, Post Traumatic Stress Disorder and Borderline Personality.

For example, Tellegen, et al. (2003) eliminated the Hysteria scale and replaced it with the RC 3 Cynicism scale. Dahlstrom, Welsh, and Dahlstrom (1972) stated that the items on the Hysteria scale seem mutually contradictory. The Hysteria scale has such seemingly unrelated issues such as somatic complaints, naiveté, denial of aggressive motives, unhappy home life and sexual conflicts. The RC researchers removed from the Hysteria scales the demoralization and somatic complaints items and assigned them to their own distinct scales, leaving mainly the items of naively trusting. Naiveté is an aspect of hysteria. The researchers reversed the scoring of the naiveté items and produced the RC 3 scale of Cynicism. Cynicism is neither an aspect of nor a substitute for Hysteria. The authors found that RC 3 poorly correlated with the MMPI-2 Hysteria scale; -.24 for females and -.18 for males. The RC 3 scale serves as an excellent example of the failure of the behavioral assumption that psychopathology is simply the sum of additive correlated symptoms.

The RC scales have been criticized for having less clinical sensitivity than the MMPI-2 Clinical Scales. Wallace (2005) found that the majority of client MMPI-2 profiles (56\%) had fewer scale elevations on the RC scales as compared to the MMPI-2 Clinical scales. In Nichols' (2011) review of the issue, he concluded "upon the presently available weight of the evidence, the RC scales, like the MMPI content scales, the sub- 
stantive MMPI-2-RF scales, and other content-based measures lack sensitivity and therefore may underestimate the presence, significance, and magnitude of clinical problems, a liability that may render them less than suitable for screening purposes (e.g., employment screening) for which maximal sensitivity is desirable..." (p. 16).

Sellbom, Ben-Porath, McNulty, Arbisi, and Graham (2006) felt that the demoralization items, subtle items, and $\mathrm{K}$ correction contributed substantially to elevation differences between the MMPI-2 and RC scales. Though the item diversity of clinical syndromes would contribute to greater sensitivity of the MMPI-2 over the RC scales, it is unlikely that $\mathrm{K}$ is a cause of the differences. $\mathrm{K}$ is often lower with greater psychopathology and would not be adding to the elevation.

There are currently no known published studies comparing the sensitivity of the MMPI-2 Clinical scales and the MMPI$\mathrm{RC}$ scales with a population of individuals diagnosed through the full dimension of psychopathology (psychotic, borderline and neurotic). Lanyon and Thomas (2013) did look at the MMPI-RF and Psychological Screening Inventory (PSI) noting that the 3 Higher Order (HO) scales of the MMPI-RF and the 3 core clinical scales of the PSI/PSI-2 were developed to broadly represent the 3 traditional categories of mental disorder: major psychiatric disorder ("psychotic"), general psychological distress ("neurotic"), and significant antisocial characteristics ("character disorder"). Their research found support for using these basic personality organizations, but the authors did not compare the sensitivity of these tests to these categories.

Gordon and Bornstein (2012) developed the Psychodiagnostic Chart (PDC) $)^{1,2}$ to operationalize the Psychodynamic Diagnostic Manual (2006). The first axis of the PDC measures personality organization with the Overall Personality Organization scale (OPO), which helps categorized individuals as mainly operating on a psychotic, borderline or neurotic to healthy level (Bornstein \& Gordon, 2012). A preliminary study of the OPO scale and PDC indicted good reliability and validity (Gordon \& Stoffey, 2013).

\section{Method}

\section{Participants}

Thirty-eight psychologists who are MMPI-2 experts were contacted by email and asked to rate their last ten psychotherapy patients, disability or forensic clients on the PDC. They were advised to share no other identifying data other than the client's initials, gender, ethnicity, age and years of education. They were given a manual as to how to use the Psychodiagnostic Chart (PDC) and asked to rate each client without looking at their MMPI-2s. Of the 38 psychologists, 15 sent in 98 PDCs with MMPI-2s. The overall sample of 104 clients consisted of 43 women, 61 men $(93 \%$ Caucasian, mean age $=40.65$, age range: $18-74$ years, mean years of education $=15.54$, education range: 6 - 22 years). The client sample included people from forensic, disability and psychotherapy evaluations, repre-

${ }^{1}$ Parts of these findings were presented at the American Psychoanalytic Association National Meeting at New York Discussion on January 17, 2013, "Research in Psychoanalysis: Creating the Psychodynamic Diagnostic Manual, Version 2 (PDM-2): Conceptual and Empirical Issues." The session was co-organized by the American Psychoanalytic Association and the Psychodynamic Psychoanalytic Research Society.

${ }^{2}$ For free copies of the PDC search online for "Psychodiagnostic Chart" or email rmgordonphd@gmail.com. senting a wide range from psychotic to neurotic personality organizations.

\section{Results}

\section{Hypothesis One}

For Hypothesis 1, we predicted that the MMPI-2 scales should show more clinical sensitivity than the RC scales at: 1) all levels of psychopathology and 2) particularly at the less pathological levels. To test this hypothesis, we calculated a series of paired-samples $t$ tests using $8 \mathrm{MMPI}-2$ scale scores (Hs, D, Hy, Pd, Pa, Pt, Sc, Ma) and 8 corresponding MMPI-RC scale scores (RC 1, RC 2, RC 3, RC 4, RC 6, RC 7, RC 8, RC 9) within each of the three levels or categories of the Overall Personality Organization (OPO) scale. The categories were derived by dividing the 10-point OPO scale into psychotic (ratings $1-3$, $\mathrm{n}=13)$, borderline $(4-6, \mathrm{n}=51)$, and neurotic $(7-10, \mathrm{n}=33)$ levels. (There were no "healthy" levels of individuals in this sample so this category was labeled "neurotic.").

Within the psychotic level, the first 3 paired-samples $t$ tests comparing MMPI-2 Hs, D, and Hy scales with the corresponding MMPI RC 1, RC 2, RC 3 scales were not significant. The paired-samples $t$ tests comparing MMPI- $2 \mathrm{Pd}, \mathrm{Pa}, \mathrm{Pt}$, and $\mathrm{Sc}$ with the MMPI RC 4, RC 6, RC 7, and RC 8 were all significant. Finally, the paired-samples $t$ test comparing the MMPI-2 Ma scale with the MMPI RC 9 scale failed to reach significance. Taken together, within the psychotic level of the OPO, $50 \%$ of the paired-samples $t$ tests were significant in the predicted direction (see Table 1 for a summary of the scale means, standard deviations, $t$ values, $p$ values and $d$ values within the psychotic level of the OPO).

Within the borderline level, the first paired-sample $t$ test comparing the MMPI-2 Hs scale with the MMPI RC 1 scale was not significant. The paired-samples $t$ tests comparing

Table 1.

The MMPI-2 vs. RC sensitivity at psychotic level of overall personality organization.

\begin{tabular}{lccccc}
\hline \multicolumn{1}{c}{ Scale } & $M$ & $S D$ & $t$ & $p$ & $d$ \\
\hline Hs & 65.69 & 17.12 & -1.95 & .075 & -.54 \\
RC 1 & 69.31 & 19.31 & & & \\
D & 71.23 & 17.54 & 1.29 & .223 & .36 \\
RC 2 & 67.38 & 18.87 & & & \\
Hy & 72.69 & 18.46 & 1.91 & .08 & .53 \\
RC 3 & 60 & 9.8 & & & \\
Pd & 79.46 & 12.07 & 7.4 & .001 & 2.06 \\
RC 4 & 56.54 & 5.75 & & & \\
Pa & 80.46 & 18.67 & 3.99 & .002 & 1.11 \\
RC 6 & 66.08 & 15.07 & & & \\
Pt & 78 & 19.43 & 6.27 & .001 & 1.74 \\
RC 7 & 54.38 & 10.28 & & & \\
Sc & 85.77 & 19.55 & 4.4 & .001 & 1.22 \\
RC 8 & 67.54 & 16.88 & & & \\
Ma & 61.38 & 14.06 & -.318 & .756 & \\
RC 9 & 62.23 & 10.13 & & & \\
\hline
\end{tabular}


MMPI-2 D, Hy, Pd, Pa, Pt, and Sc with the corresponding MMPI RC 2, RC 3, RC 4, RC 6, RC 7, and RC 8 were all significant.

The final paired-sample $t$ test comparing the MMPI-2 Ma scale with the MMPI RC 9 scale failed to reach significance. Taken together, $75 \%$ of the paired-sample $t$ tests within the borderline level were significant in the predicted direction (see Table 2 for a summary of the scale means, standard deviations, $t$ values, $p$ values and $d$ values within the borderline level of the $\mathrm{OPO})$.

The last set of paired-sample $t$ tests examined mean differences between the MMPI-2 and MMPI RC scales within the neurotic level of the OPO. The only paired-samples $t$ test that failed to reach significance was between the MMPI-2 D and MMPI RC 2 scale. Within the neurotic level, a total of $88 \%$ of the paired-samples $t$ tests were significant in the predicted direction (see Table 3 for a summary of the scale means, scale standard deviations, $t$ values, $p$ values and $d$ values within the neurotic level of the OPO).

Across all three levels of the Overall Personality Organization (OPO) scale a total of $71 \%$ of the paired-samples $t$ tests were significant. Taken together, the above analyses lend strong support to Hypothesis 1 (a). In support of Hypothesis 1 (b), the neurotic level of the OPO contained the largest percentage of significant pair-sample $t$ tests $(88 \%)$, followed by the borderline level $(75 \%)$, and lastly the psychotic level (50\%).

\section{Hypothesis Two}

For Hypothesis 2, we predicted that the added $\mathrm{K}$ correction does not account for the differences in elevation between the MMPI-2 scales versus the MMPI RC scales. To test this hypothesis, we calculated means and standard deviations both within each level of the Overall Personality Organization (OPO) scale and an overall mean and standard deviation across all three levels of the OPO. The level means and standard deviations were: psychotic $(\mathrm{M}=40.08, \mathrm{SD}=7.16)$, borderline $(\mathrm{M}=$ $48.44, \mathrm{SD}=8.65)$ and neurotic $(\mathrm{M}=55.88, \mathrm{SD}=9.33)$. The

Table 2.

The MMPI-2 vs. RC sensitivity borderline level of the overall personality organization.

\begin{tabular}{lccccc}
\hline \multicolumn{1}{c}{ Scale } & $M$ & $S D$ & $t$ & $p$ & $d$ \\
\hline Hs & 58.86 & 13.36 & 1.34 & .185 & .19 \\
RC 1 & 57.63 & 13.98 & & & \\
D & 66.57 & 16.01 & 3.43 & .001 & .48 \\
RC 2 & 61.94 & 15.91 & & & \\
Hy & 64.31 & 13.98 & 5.23 & .001 & .73 \\
RC 3 & 50.24 & 10.13 & & & \\
Pd & 66.06 & 11.2 & 7.15 & .001 & 1 \\
RC 4 & 54.04 & 10.13 & & & \\
Pa & 63.33 & 11.71 & 5.6 & .001 & .78 \\
RC 6 & 53.94 & 11.9 & & & \\
Pt & 63.67 & 12.98 & 7.69 & .001 & 1.08 \\
RC 7 & 50.86 & 10.98 & & & \\
Sc & 62.08 & 12.39 & 6.02 & .001 & .84 \\
RC 8 & 52.86 & 11.78 & & & \\
Ma & 50.63 & 8.57 & 1.21 & .234 & .17 \\
RC 9 & 49.35 & 8.31 & & & \\
\hline
\end{tabular}

Table 3.

The MMPI-2 vs. RC sensitivity neurotic level of the overall personality organization.

\begin{tabular}{lccccc}
\hline \multicolumn{1}{c}{ Scale } & $M$ & $S D$ & $t$ & $p$ & $d$ \\
\hline Hs & 56.67 & 11.24 & 3.21 & .003 & .56 \\
RC 1 & 52.85 & 11.55 & & & \\
D & 54.73 & 9.92 & 1.1 & .279 & .19 \\
RC 2 & 52.85 & 7.44 & & & \\
Hy & 59.85 & 12.15 & 4.49 & .001 & .78 \\
RC 3 & 46.79 & 8.31 & & & \\
Pd & 59.7 & 8.87 & 4.41 & .001 & .77 \\
RC 4 & 52 & 9.68 & & & \\
Pa & 56.97 & 9.85 & 5.9 & .001 & 1.03 \\
RC 6 & 46.48 & 8.02 & & & \\
Pt & 57.27 & 10.07 & 6.15 & .001 & 1.07 \\
RC 7 & 45.97 & 8.04 & & & \\
Sc & 56.18 & 9.28 & 4.91 & .001 & .85 \\
RC 8 & 45.91 & 7.84 & & & \\
Ma & 49.24 & 6.49 & 2.67 & .012 & .46 \\
RC 9 & 45.76 & 6.76 & & & \\
\hline
\end{tabular}

overall mean and standard deviation across all levels of the OPO was $\mathrm{M}=49.80$ and $\mathrm{SD}=10.30$ indicating that the greater elevations in the MMPI-2 Clinical scales are not due to the $\mathrm{K}$ correction.

\section{Hypothesis Three}

For hypothesis 3, we predicted that for each clinical scale, the RC scale adds very little incremental validity to the MMPI2 clinical scales in predicting the level of severity in the Overall Personality Organization (OPO) scale. In testing hypothesis 3, a series of multiple regressions were conducted where an MMPI2 scale was entered first, followed by its corresponding RC scale.

In the first regression, Hs was entered first followed by RC 1. For the first model, Hs accounted for $3.80 \%$ of the variance in the OPO scale $\left(\mathrm{R}^{2}=.038\right)$. When $\mathrm{RC} 1$ is included (model 2$)$, this value increases to .149 or $14.9 \%$ of the variance in the OPO scale $\left(\mathrm{R}^{2}=.149\right)$, an increase of $11.1 \%$. The $\mathrm{F}$ change for model 2 was significant, $\mathrm{F}(1,94)=12.303, p=.001$, indicating that adding RC 1 significantly improved the prediction of the OPO scale compared to using Hs as a single predictor.

In the second regression, D was entered first followed by RC 2. For model $1, \mathrm{D}$ accounted for $15 \%$ of the variance in the OPO scale $\left(\mathrm{R}^{2}=.150\right)$. When $\mathrm{RC} 2$ was entered in model 2 , this value increased to .153 or $15.3 \%\left(\mathrm{R}^{2}=.153\right)$, an increase of only $.30 \%$. The $\mathrm{F}$ change for model 2 was not significant, $\mathrm{F}(1,94)=.382, p=.538$, indicating that adding the RC 2 scale did not significantly improve the prediction of the OPO scale compared to using only D.

The third regression entered Hy followed by RC 3. For model 1 , Hy accounted for $7.30 \%$ of the variance in the OPO scale $\left(\mathrm{R}^{2}=.073\right)$. For model 2 , this value increased to .247 or $24.7 \%\left(\mathrm{R}^{2}=.247\right)$, an increase of $17.4 \%$. The $\mathrm{F}$ change was significant, $\mathrm{F}(1,94)=21.68, p=.001$, indicating the adding $\mathrm{RC}$ 3 did significantly improve the prediction of the OPO scale above and beyond what was predicted using only Hy. However, 
RC 3 is a measure of Cynicism and not hysterical personality traits and therefore cannot be considered a substitute for the MMPI-2 Hy scale.

In the next regression, model 1 contained $\mathrm{Pd}$, with $\mathrm{RC} 4$ added in model 2 . For model 1, Pd accounted for $23.9 \%$ of the variance in the OPO scale $\left(\mathrm{R}^{2}=.239\right)$. For model 2 , this value increased to .241 or $24.1 \%\left(\mathrm{R}^{2}=.241\right)$, an increase of only $.2 \%$. This change in $\mathrm{R}^{2}$ produced a non-significant $\mathrm{F}(1,94)=.249, p$ $=.619$, indicating no predictive improvement with the inclusion of RC 4 .

The fifth regression entered $\mathrm{Pa}$ followed by $\mathrm{RC} 6$. For the first model with $\mathrm{Pa}$ entered alone, $\mathrm{Pa}$ accounted for $24.2 \%$ of the variance in the OPO scale $\left(\mathrm{R}^{2}=.242\right)$. When $\mathrm{RC} 6$ was added in model 2 , this value increased to .289 or $28.9 \%$, producing a significant $\mathrm{F}(1,94)=6.228, p=.014$, indicating improvement in predicting the OPO scale compared to using only $\mathrm{Pa}$. An examination of the beta values for these two predictors in model 2 suggests, however, that $\mathrm{Pa}$ predicts more of the variance in the OPO scale than does RC 6 (-.317 vs. -.279). That is, while RC 6 predicts additional variance above and beyond that predicted by $\mathrm{Pa}$ alone, the amount predicted is small relative to $\mathrm{Pa}$.

We next entered Pt in model 1 followed by RC 7 in model 2. For model 1, Pt accounted for $18.2 \%$ of the variance in the OPO scale $\left(\mathrm{R}^{2}=.182\right)$. For model 2 , this value increased to .184 or $18.4\left(\mathrm{R}^{2}=.184\right)$, an increase of only $.2 \%$. This change in $\mathrm{R}^{2}$ produced a non-significant $\mathrm{F}(1,94)=.306, p$ $=.582$, indicating no predictive improvement with the inclusion of RC 7.

The next regression entered Sc in model 1 and RC 8 in model 2. For model 1, Sc accounted for $28.3 \%$ of the variance in the OPO scale $\left(\mathrm{R}^{2}=.283\right)$. For model 2 , this value increased to .320 or $32.0 .1 \%\left(\mathrm{R}^{2}=.320\right)$, an increase of $3.7 \%$. This change in $\mathrm{R}^{2}$ produced a significant $\mathrm{F}(1,94)=5.036, p=.027$, indicating improvement in predicting in the OPO scale with the inclusion of RC 8 . However, the beta values indicate the $\mathrm{Sc}$ predicts more of the variance in the OPO scale than does the RC 8 scale (-.366 vs. -.253$)$.

In the final regression, Ma was entered first, followed in model 2 with RC 9. For model 1, Ma accounted for $11.3 \%$ of the variance in the OPO scale $\left(\mathrm{R}^{2}=.113\right)$. Adding $\mathrm{RC} 9$ in model two increased this value to $24.1 \%\left(\mathrm{R}^{2}=.241\right)$, producing a significant $\mathrm{F}(1,94)=15.809, p=.001$. In this regression, the beta values indicate that RC 9 in comparison to MA also predicts more of the variance in the OPO scale (-.476 vs. -.021$)$.

In summary, 5 of the 8 RC scales (RC 1, RC 2, RC 6, RC 8, and $\mathrm{RC} 9$ ) added incremental validity to the MMPI-2 clinical scales in predicting the level of severity in the Overall Personality Organization (OPO) scale. However, an examination of the beta values indicates that for 2 of the 5 RC scales (RC 6 and $\mathrm{RC} 8$ ) the amount of additional predicted variance is relatively small in comparison to the amount predicted by their corresponding MMPI-2 scales ( $\mathrm{Pa}$ and SC). Taken together, in terms of predictive utility, the results suggest that only 3 of the 8 or $37.5 \%$ of the RC scales contribute incrementally in predicting the level of severity in the OPO scale, lending support to hypothesis 3 .

\section{Discussion}

This study looked at the clinical sensitivity of the MMPI-2 Clinical scales vs. the MMPI-RC scales with 98 clients from forensic, disability and psychotherapy evaluations, representing a wide range from psychotic, borderline to neurotic levels of personality organization as measured by the Psychodiagnostic Chart. Since the RC scales lack a diversity of items and subtly, we hypothesized that the MMPI-2 Clinical scales would have more clinical sensitivity than the RC scales at: 1) all levels of psychopathology and 2) particularly at the less pathological levels where subtlety is more of an issue in detecting psychopathology.

We found that the clients at the psychotic level of personality organization as measured by the PDC, the MMPI-2 Hs, D, Hy and Ma scales were not significantly different than the corresponding MMPI RC 1, RC 2, RC 3, RC 9 scales. The MMPI-2 $\mathrm{Pd}, \mathrm{Pa}, \mathrm{Pt}$, and $\mathrm{Sc}$ were all significantly higher than the corresponding RC 4, RC 6, RC 7, and RC 8. Only 4 of the RC scales reached clinical significance (T 65 or more), while 7 of the 8 MMPI 2 Clinical scales were $\mathrm{T} 65$ or more for clients at the psychotic level.

For those clients rated on the PDC at the borderline level the MMPI-2, Hs and Ma scales and the RC 1 and RC 9 scales were not significantly different. The MMPI-2 D, Hy, Pd, Pa, Pt, and Sc were all significantly higher than the corresponding $\mathrm{RC} 2$, RC 3, RC 4, RC 6, RC 7, and RC 8 scales. D and Pd were in the clinical range, but none of the RC scales reached the clinical range of $\mathrm{T} 65$.

For those clients rated on the PDC at the neurotic level, only the MMPI-2 D scale and the RC 2 scales were not significantly different. The MMPI-2 Hs, Hy, Pd, Pa, Pt, Sc and Ma were all significantly higher than the corresponding RC scales. Across all three levels of the Overall Personality Organization (OPO), the neurotic level of the OPO had $88 \%$ difference between the MMPI-2 and RC, followed by the borderline level (75\%) and lastly the psychotic level (50\%).

The added K correction to the MMPI-2 Clinical scales does not seem likely to account for the differences in elevations. Overall, $\mathrm{K}$ was at the normal level $(\mathrm{M}=49.80)$. K was lowest in the psychotic level and highest in the neurotic level. $\mathrm{K}$ functions in much the same way as the subtle items function in those scales that are not $\mathrm{K}$ corrected, i.e. as the defensive aspects of the psychopathology (Gordon, 1989). These diagnostically valid items are not artificially elevating the Clinical scales. Both $\mathrm{K}$ and the subtle items add diagnostic sensitivity to the MMPI-2 scales not found in homogenized scales.

Most of the RC scales add little to no incremental validity to the MMPI-2 Clinical scales in predicting the level of severity in the Overall Personality Organization (OPO) scale. However, $\mathrm{RC} 1, \mathrm{RC} 2$, and RC 9 do have value in adding to the incremental validity of the MMPI-2 and should be considered as supplemental scales. We recommend using the MMPI-2 Clinical scales for screening psychopathology and to use the RC scales only as supplemental scales. The broader issue, however, is the value of criteria-based scales. The MMPI-2 scales were based on the complexity expressed in the conflicts of disturbed individuals. The RC scales are based on behavioral reductionistic assumptions of psychopathology which fail to capture the complexity of human conflicts.

\section{REFERENCES}

Binford, A., \& Liljequist, L. (2008). Behavioral correlates of selected MMPI-2 clinical, content, and restructured clinical scales. Journal of Personality Assessment, 90, 608-614. 


\section{R. M. GORDON ET AL.}

doi:10.1080/00223890802388657

Bolinskey, P. K., \& Nichols, D. S. (2011). Construct drift in the MMPI2 restructured clinical scales: Further evidence and a possible historic example. Journal of Clinical Psychology, 67, 907-917. doi: $10.1002 /$ jclp. 20814

Bornstein, R. F., \& Gordon, R. M. (2012). What do practitioners want in a diagnostic taxonomy? Comparing the PDM with DSM and ICD. Division/Review: A Quarterly Psychoanalytic Forum, 6.

Dahlstrom, W. G., Welsh, G. S., \& Dahlstrom, L. E. (1972). An MMPI handbook: Vol. I: Clinical interpretations. Minneapolis, MN: University of Minnesota Press.

Gordon, R. M. (1989). Interpreting MMPI subtle scales as representing defense mechanisms. Paper presented at the 24th Annual Symposium on Recent Developments in the Use of the MMPI, Honolulu.

Gordon, R. M. (2006). False assumptions about psychopathology, hysteria and the MMPI-2 restructured clinical scales. Psychological Reports, 98, 870-872. doi:10.2466/pr0.98.3.870-872

Gordon, R. M., \& Stoffey, R. W. (2013). Research in psychoanalysis: Creating the psychodynamic diagnostic manual, version 2 (PDM-2): Conceptual and empirical issues. Presented at the American Psychoanalytic Association National Meeting, New York.

Lanyon, R. I., \& Thomas, M. L. (2013). Assessment of global psychiatric categories: The PSI/PSI-2 and the MMPI-2-RF. Psychological Assessment, 25, 227-232. doi:10.1037/a0030313
Nichols, D. S. (2011). Essentials of MMPI-2 assessment (2nd ed.). Essentials of psychological assessment. Hoboken, NJ: John Wiley \& Sons Inc.

PDM Task Force (2006). Psychodynamic diagnostic manual. Silver Spring, MD: Alliance of Psychoanalytic Organizations.

Rouse, S. V., Greene, R. L., Butcher, J. N., Nichols, D. S., \& Williams, C. L. (2008). What do the MMPI-2 restructured clinical scales reliably measure? Answers from multiple research settings. Journal of Personality Assessment, 90, 435-442. doi: $10.1080 / 00223890802248695$

Sellbom, M., Ben-Porath, Y. S., McNulty, J. L., Arbisi, P. A., \& Graham, J. R. (2006). Elevation differences between MMPI-2 clinical and restructured clinical (RC) scales: Frequency, origins, and interpretative implications. Assessment, 13, 430-441. doi:10.1177/1073191106293349

Tellegen, A., Ben-Porath, Y. S., McNulty, J. L., Arbisi, P. A., Graham, J. R., \& Kaemmer, B. (2003). MMPI-2 restructured clinical (RC) scales: Development, validation, and interpretation. Minneapolis, MN: University of Minnesota Press.

Wallace, A., \& Laura, L. (2005). A comparison of the correlational structures and elevation patterns of the MMPI-2 restructured clinical (RC) and clinical scales. Assessment, 12, 290-294.

doi: $10.1177 / 1073191105276250$ 